\title{
Spectroscopic observations of southern Galactic planetary nebulae
}

\author{
Slawomir K. Górny \\ N. Copernicus Astronomical Center, Toruń, Poland
}

\begin{abstract}
We present preliminary results from low resolution spectroscopic observations of planetary nebulae located in the southern Milky Way disk.
\end{abstract}

Keywords. planetary nebulae: general, techniques: spectroscopic

We have observed 50 planetary nebulae (PNe) located between Vela and Norma constellations. The list of their names and PNG numbers is given in Table 1. Spectra of all objects were secured with the 1.9-meter telescope at the South African Astronomical Observatory in 2003-2006 as a subsidiary program during observing runs dedicated to the search for new PNe towards the Galactic bulge (Górny 2006). The usual spectral coverage was 3500 - $7400 \AA$ with an average resolution of 1000 . The total typical integration time was 30 to 60 minutes divided into sub-exposures to secure unsaturated measurements of the strongest lines.

Procedures from the long-slit spectral package of MIDAS, developed by the European Southern Observatory, were used to reduce and calibrate the spectra. Nebular line intensities were measured from 1D spectra by employing the REWIA package developed by J.Borkowski (NCAC) adopting Gaussian profiles and performing multi-Gaussian fits when necessary.

Table 1. List of observed PNe.

\begin{tabular}{|c|c|c|c|}
\hline $268.4+02.4$ & PB 5 & $307.3+05.0$ & Wray $16-128$ \\
\hline 269.7-03.6 & PB 3 & $308.2+07.7$ & MeWe 1-3 \\
\hline $279.6-03.1$ & He $2-36$ & $309.0+00.8$ & He $2-96$ \\
\hline $283.8+02.2$ & Мy 60 & $310.7-02.9$ & He $2-103$ \\
\hline $283.8-04.2$ & He 2-39 & $311.4+02.8$ & He 2-102 \\
\hline $285.4+01.5$ & Pe 1-1 & $312.6-01.8$ & He $2-107$ \\
\hline $285.7+01.2$ & Pe 1-2 & $314.4+02.2$ & PM 1-81 \\
\hline $291.4+19.2$ & ESO $320-28$ & $315.1-13.0$ & He 2-131 \\
\hline $292.8+01.1$ & He $2-67$ & $315.4+05.2$ & Не 2-109 \\
\hline 293.1-00.0 & BMPJ1128-61 & $316.1+08.4$ & He $2-108$ \\
\hline $293.6+01.2$ & He $2-70$ & $318.3-02.5$ & He 2-116 \\
\hline $294.6+04.7$ & NGC 3918 & $319.2+06.8$ & He 2-112 \\
\hline 294.9-04.3 & Hе 2-68 & $320.9+02.0$ & Не $2-117$ \\
\hline $295.3-09.3$ & He 2-62 & $321.3+02.8$ & He $2-115$ \\
\hline 296.3-03.0 & He $2-73$ & $321.8+01.9$ & He $2-120$ \\
\hline $297.4+03.7$ & He $2-78$ & $322.5-05.2$ & NGC 5979 \\
\hline $299.0+18.4$ & K 1-23 & $323.9+02.4$ & He $2-123$ \\
\hline $299.5+02.4$ & He $2-82$ & $324.2+02.5$ & He $2-125$ \\
\hline $300.2+00.6$ & He $2-83$ & $324.8-01.1$ & He $2-133$ \\
\hline $300.4-00.9$ & He 2-84 & $327.1-01.8$ & He $2-140$ \\
\hline $300.5-01.1$ & He $2-85$ & $327.8-01$ & He $2-143$ \\
\hline $300.7-02.0$ & He $2-86$ & $330.7+04.1$ & Cn 1-1 \\
\hline $300.8-03.4$ & ESO $095-12$ & $330.9+04.3$ & Wray $16-189$ \\
\hline $304.5-04.8$ & IC 4119 & $336.9+08.3$ & StWr 1 \\
\hline $305.1+01.4$ & He $2-90$ & $338.1-08.3$ & NGC 6326 \\
\hline
\end{tabular}




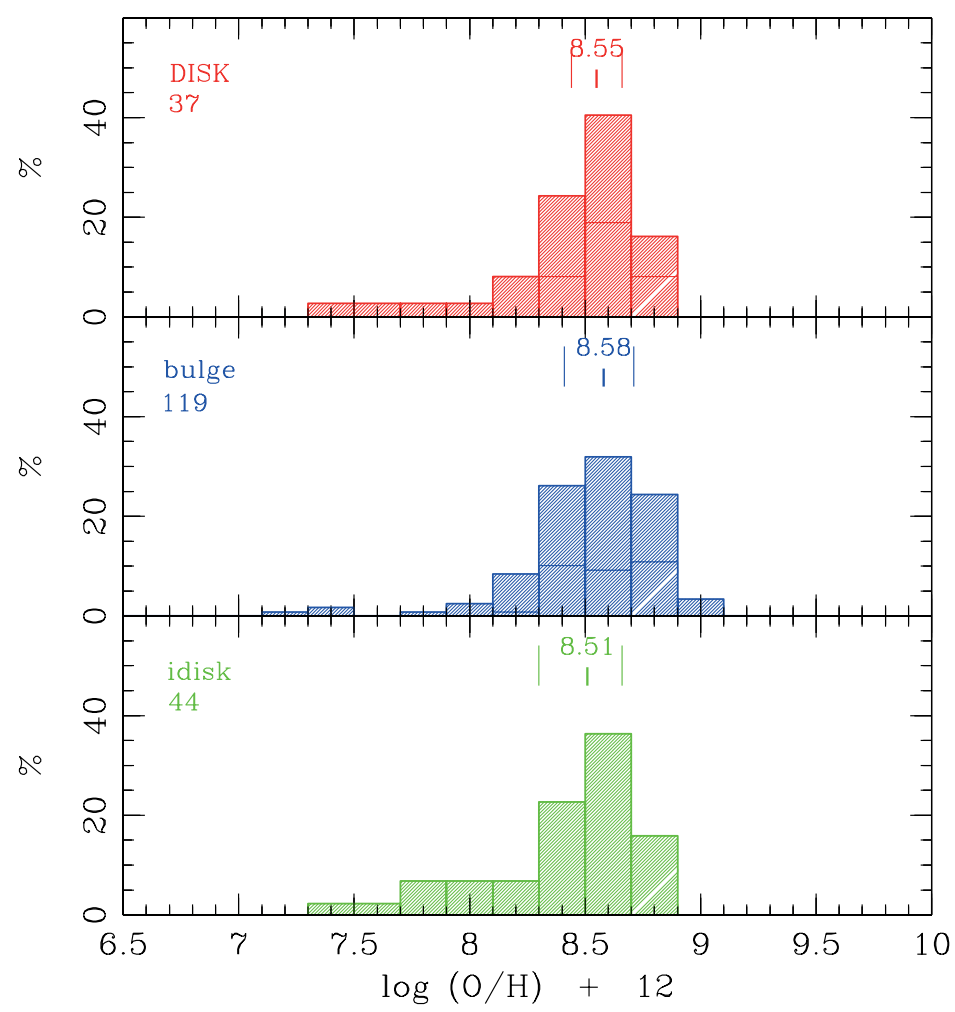

Figure 1. Histograms of the $\mathrm{O} / \mathrm{H}$ nebular ratio for Galactic disk (top), bulge (middle) and inner-disk (bottom) PNe samples. The median values of the distributions, the 25 and 75 percentiles and the number of objects with available data used in each panel are also shown.

We used the classical empirical method to derive plasma parameters and chemical abundances of helium and nitrogen as well as of oxygen, argon, neon, sulfur and chlorine deriving them with the code ABELION as in Górny et al. (2009).

The determined distribution of nebular $\mathrm{O} / \mathrm{H}$ ratio for the observed sample of $\mathrm{PNe}$ is presented in the top panel of Fig. 1. It can be compared with the distributions derived for the samples of Galactic bulge and inner-disk PNe using data from Górny et al. (2009). No statistically meaningful differences for $\mathrm{O} / \mathrm{H}$ ratio can be noticed in Fig. 1 between these three groups of PNe leading to the conclusion that the gradient of chemical abundances must be almost flat in the Galaxy within the Sun orbit.

\section{Acknowledgements}

This work was supported from grant N N203 511838 of the Science and High Education Ministry of Poland.

\section{References}

Górny, S. K. 2007, in: M. J. Barlow \& R. H. Mendez, (eds.) IAU Symp. 234, Planetary Nebulae in our Galaxy and Beyond, (Cambridge: CUP), p. 409

Górny, S. K., Chiappini, C., Stasińska, G., \& Cuisinier, F. 2009, A\&A, 500, 1089 